\title{
Omni-directional wind-driven triboelectric nanogenerator with cross-shaped dielectric film
}

\author{
Yoseop Shin, Sungjun Cho, Sejin Han and Gun Young Jung (i)
}

\begin{abstract}
Triboelectric nanogenerators (TENGs) are actively being researched and developed to become a new external power unit for various electronics and applications. Wind is proposed as a mechanical energy source to flutter the dielectric film in wind-driven TENGs as it is clean, abundant, ubiquitous, and sustainable. Herein, we propose a TENG structure with dielectric films bent in four directions to collect the wind energy supply from all directions, unlike the conventional wind-driven TENGs which can only harvest the wind energy from one direction. Aluminum (Al) layer was intercalated within the dielectric film to improve electrostatic induction, resulting in improved triboelectric performances. Maximum open-circuit voltage $\left(\mathrm{V}_{\text {oc }}\right)$ of $233 \mathrm{~V}$, short-circuit current $\left(\mathrm{I}_{\mathrm{sc}}\right)$ of $348 \mu \mathrm{A}$, and output power density of $46.1 \mathrm{~W}$ $\mathrm{m}^{-2}$ at an external load of $1 \mathrm{M} \Omega$ under a wind speed of $9 \mathrm{~m} \mathrm{~s}^{-1}$ were revealed, and it faithfully lit "LED" characters composed of 25 LEDs.
\end{abstract}

Keywords: Energy harvesting, Triboelectric nanogenerator, Wind energy, Electrification, Electrostatic induction

\section{Introduction}

With the advent of the fourth industrial revolution, the Internet of Things (IoT) technology that allows data to be sent and received in real time by attaching sensors to objects is developing rapidly [1-3]. Wearable devices that are directly related to IoT are also being rapidly commercialized [4-6] and are attached close to the body of users in the forms of glasses [7], wristwatches [8], and shoes [9] for data collection from the surroundings. Therefore, the wearables must be small, light, and wireless [10]. Lithium-ion batteries are normally used as an external power source to operate these sensors and portable devices [11], but have the disadvantages of recharging requirement, replacement due to the limited lifetime and power capacity, and the risk of explosion [10, 12]. Therefore, development of new power supplies that can overcome the limitations of lithium-ion batteries is essential.

*Correspondence: gyjung@gist.ac.kr

'Yoseop Shina and Sungjun Cho equally contributed

School of Materials Science and Engineering, Gwangju Institute

of Science and Technology (GIST), 123 Cheomdan-gwagiro, Buk-gu,

Gwangju 61005, Republic of Korea
Currently, a variety of wireless sensors using IoT are ubiquitously utilized in military [13], industrial [14], academic [15], and leisure [16] fields, rendering a growing interest in permanent energy harvesting, in which the wasted and natural energies can be collected and utilized from the surrounding environment. Energy harvesting technologies convert heat [17], light [18], and mechanical energy [19] into electrical energy. Today, solar cells are one of the representative energy harvesting devices [20] using the photoelectric effect theory. However, the solar cells have drawbacks such as high weather-dependence and geographical limitations. New energy harvesting devices are required to alternate the solar cells, giving rise to interest in mechanical energy harvesting.

Two approaches for the mechanical energy harvesting, which can be generated by various human movements [21] and wind [22], and piezoelectric [23] and triboelectric [24] effects. Piezoelectric nanogenerators (PENGs) generate electrical signals upon receiving external stress or vibration $[9,25-28]$. Triboelectric nanogenerators (TENGs) use an electrostatic phenomenon generated by the contact of two different materials, leading to a potential difference between the materials [29-31]. Various 
triboelectric nanogenerators have been reported using human mechanical stimuli and various natural energies, such as wind, ultrasonic, raindrops, and water waves [22]. Wind has recently been utilized as a mechanical energy source to flutter the dielectric film for wind-driven TENGs because wind is clean, abundant, ubiquitous, and sustainable [32-34]. The wind-driven TENGs have advantages of simple manufacturing at low cost, low weight, and no explosion risk compared to the lithiumion batteries [35-38].

Interest in mobility is growing in modern era; for example, drones with numerous wireless sensors are used in a wide variety of fields, including the observation of activities, video filming, and offline delivery [39-41]. Accordingly, an auxiliary power supply providing sustainable energy is required to increase the limited flight time of drones, which are indispensable in the fourth industrial age [42].

Herein, we propose a wind-driven TENG structure with a cross-shaped dielectric film bent in four directions (C-TENG), which can produce a suitable external power supply in all wind directions, unlike the vehicles or subways that can run in only one direction. The TENG could efficiently harvest wind energy from all direction, suitable for drones flying in arbitrary directions. Additionally, another TENG, in which an Al layer is intercalated within a polytetrafluoroethylene (PTFE) film (CIA-TENG), was produced to improve electrostatic induction, resulting in improved triboelectric performances. $\mathrm{Al}$ and PTFE were selected as the triboelectric materials. The triboelectric performances of C-TENG and CIA-TENG were compared in this study. The effect of wind direction on the triboelectric performances was also tested, demonstrating that the wind energy can be harvested omnidirectionally.

\section{Experimental details}

\subsection{C-film and CIA-film fabrication}

A PTFE film tape $\left(3 \mathrm{M}^{\mathrm{TM}}\right.$, thickness of $\left.50 \mu \mathrm{m}\right)$ was used as a triboelectric material. The $\mathrm{C}$-film was produced by sticking two PTFE film tapes each other and cutting them in the form of a cross-shape. The central square area of the C-film is $2 \times 2 \mathrm{~cm}^{2}$. An Al foil (HANSUNG, thickness of $15 \mu \mathrm{m}$ ) was used for the intercalated metal layer within the PTFE film. The CIA-film was produced by attaching the two PTFE tapes to both sides of the $\mathrm{Al}$ foil. The $\mathrm{Al}$ foil was protruded $5 \mathrm{~mm}$ from the PTFE film for the connection to the Al bottom electrode electrically. Dry etching was performed using a reactive ion echer (Plasmart, MINIPLASMA station) under following conditions; $\mathrm{O}_{2}$, $\mathrm{CF}_{4}$, and Ar gases (10, 30, and $15 \mathrm{sccm}$, respectively), 20 mTorr, and $400 \mathrm{~W}$ for $60 \mathrm{~s}$.

\subsection{CIA-TENG fabrication}

The $\mathrm{Al}$ tape (DUCSUNG HITECH, thickness of $50 \mu \mathrm{m}$ ) was used both top and bottom electrodes as well. After bending the cross-shaped CIA-film in four directions, the $\mathrm{Al}$ foil protruded from the CIA-film was electrically connected to the $\mathrm{Al}$ tape (attched to the bottom glass plate working as the bottom electrode) with a conductive carbon adhesive tape (SHILPA ENTERPRISES, thickness of $15 \mu \mathrm{m}$ ). Another Al tape (for the role of top electrode) was attached to the top glass plate with a size of $2 \times 2$ $\mathrm{cm}^{2}$.

\subsection{Triboelectric performance measurements}

An oscilloscope (Tektronix, DPD4014B) and pre-amplifier (Stanford Research, SR570) were used to measure the output voltage and current. During the measurement, wind was supplied with a commercial nitrogen blow gun, and the wind speed was measured using a commercial anemometer (Testo, Testo 417). The PTFE surface roughness was analyzed using AFM equipment (Park's system, XE-100).

\section{Results and discussion}

\subsection{Structure design and triboelectric materials for C-TENG and CIA-TENG}

Figure 1 shows the structures and photographs of two wind-driven TENGs using different dielectric films bent into four directions for pursuing the omnidirectional fluctuation by the wind. PTFE and $\mathrm{Al}$ were used as the triboelectric materials; the PTFE is a dielectric polymer composed of monomers of tetrafluoroethylene, which has abundant fluorines (F). The $\mathrm{F}$ atom has the highest electron affinity among many atoms, resulting in accumulated electron charges on the PTFE surface after touching the $\mathrm{Al}$ electrode, which is positively charged by electrification. The $\mathrm{Al}$ acts as bottom- and top electrodes as well in this study; thus, it simplifies the manufacturing process of the TENG. Inductively coupled plasma-reactive ion etching (ICP-RIE) was performed on the PTFE surface to increase the contact surface area for enhancing the triboelectric performance. The RMS roughness of the PTFE surface was measured as $35.1 \mathrm{~nm}$ (before etching) and $53.0 \mathrm{~nm}$ (after etching) at a scan area of $15 \times 15 \mu \mathrm{m}^{2}$ (Additional file 1: Fig. S1).

The C-TENG with the cross-shaped PTFE film (C-film) bent in four directions is depicted and shown in Fig. $1 \mathrm{a}$ and $\mathrm{b}$. The contact friction is generated by the wind-driven fluctuation of the C-film at the interface with the Al top electrode. The cross-shaped C-film allows the TENG to collect mechanical energy regardless of wind direction and is suitable for vehicles (e.g., drones) moving in arbitrary directions. The effective 


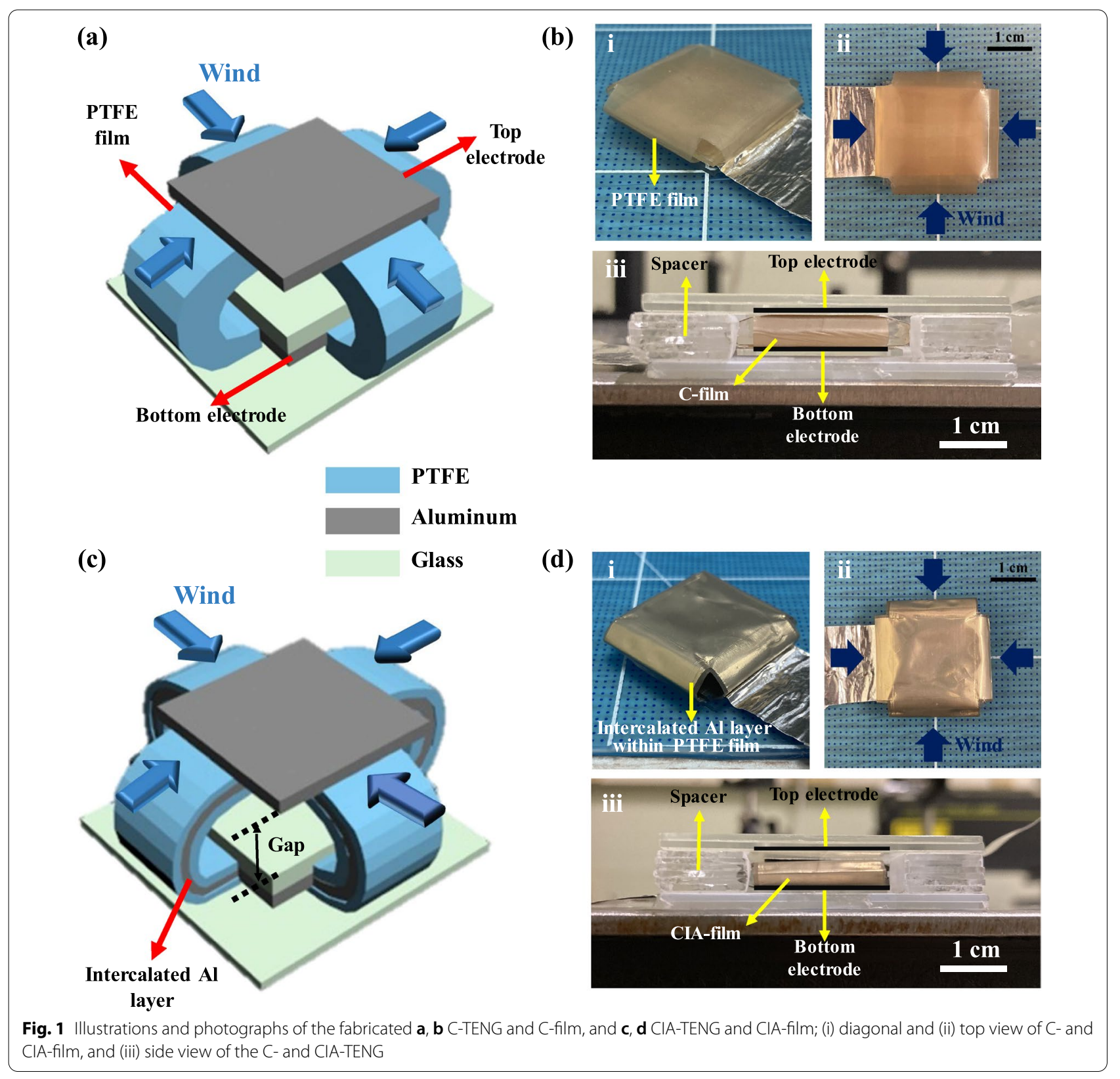

triboelectric contact area of the C-film is the center part $\left(2 \times 2 \mathrm{~cm}^{2}\right)$ that actually touches the $\mathrm{Al}$ top electrode. Figure $1 \mathrm{c}$ and $\mathrm{d}$ show another TENG with the intercalated $\mathrm{Al}$ layer within the C-film (CIA-film) that is hereafter called CIA-TENG. These two device structures are identical except for the existence of the intercalated Al layer within the PTFE dielectric film. The four wings of CIA-film were bent in four directions and electrically connected to the $\mathrm{Al}$ bottom electrode through the protruded intercalated $\mathrm{Al}$ layer. The fixed CIA-film is not free from lateral movement but has an elasticity advantageous for the up/down vertical vibration by the wind (Additional file 2: Movie S1).

\subsection{Triboelectric performance of C-TENG and CIA-TENG}

Figure 2 shows the triboelectric performances of the etched C-TENG and CIA-TENG at a wind speed of $9 \mathrm{~m} \mathrm{~s}^{-1}$. In the case of the C-TENG, a maximum opencircuit voltage $\left(\mathrm{V}_{\mathrm{oc}}\right)$ of $153 \mathrm{~V}$ and short-circuit current $\left(\mathrm{I}_{\mathrm{sc}}\right)$ of $51.8 \mu \mathrm{A}$ were measured (Fig. 2a and b). For the CIA-TENG, the maximum $\mathrm{V}_{\mathrm{oc}}$ and $\mathrm{I}_{\mathrm{sc}}$ were $233 \mathrm{~V}$ and $348 \mu \mathrm{A}$, respectively, which were 1.52 and 6.72 fold larger 
(a)

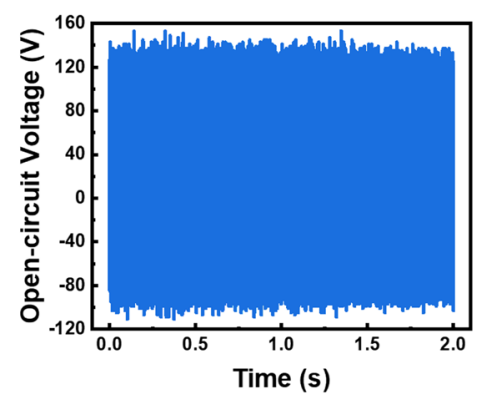

(d)

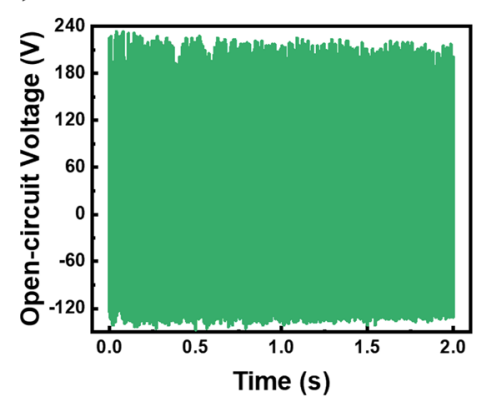

(b)

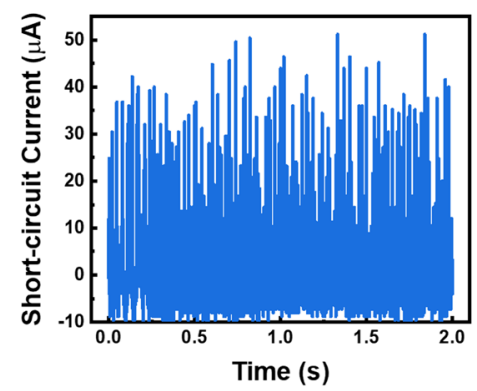

(e)

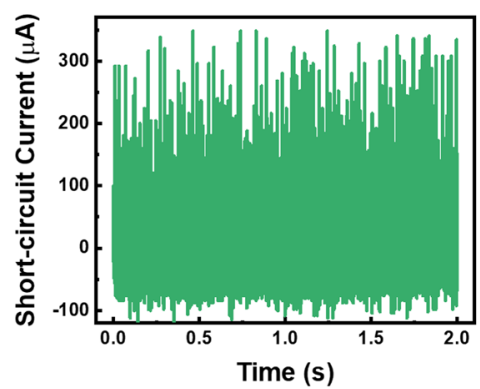

(c)

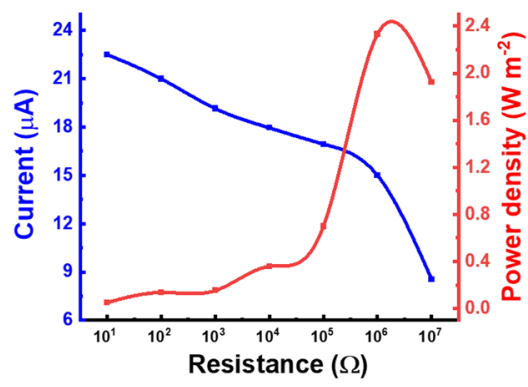

(f)

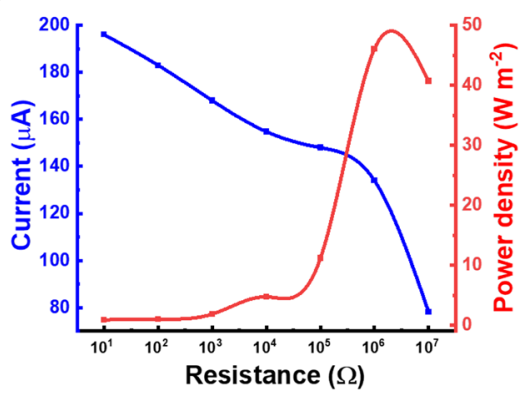

Fig. 2 Output performances of the C-TENG and CIA-TENG using the etched PTFE film at a wind speed of $9 \mathrm{~m} \mathrm{~s}^{-1}$; $\mathrm{V}_{\mathrm{OC}} \mathrm{I}_{\mathrm{sC}}$ and output power density of $\mathbf{a}-\mathbf{c} C-T E N G$, and $\mathbf{d}-\mathbf{f} C \mathrm{~A}-\mathrm{T} E N G$, respectively

than those of the C-TENG (Fig. 2d and e). External resistances to the TENGs were applied to measure the output power density. At an external resistance of $1 \mathrm{M} \Omega$, the maximum output power density of the CIA-TENG was $46.1 \mathrm{~W} \mathrm{~m}^{-2}$, which is approximately 20-fold higher than the $2.33 \mathrm{~W} \mathrm{~m}^{-2}$ of the C-TENG (Fig. 2c and $\mathrm{f}$ ). The $\mathrm{I}_{\mathrm{sc}}$ increased noticeably when using the intercalated $\mathrm{Al}$ layer. A maximum average output power derived from the root-mean-square (RMS) voltage was $2.36 \mathrm{~mW}$ at an external resistance of $1 \mathrm{M} \Omega$ in CIA-TENG (Additional file 1: Fig. S2).

To identify the effect of surface roughness, two TENGs with the unetched C-film and CIA-film were also measured. The maximum $\mathrm{V}_{\mathrm{oc}}$ and $\mathrm{I}_{\mathrm{sc}}$ of the unetched C-TENG were $104 \mathrm{~V}$ and $24.4 \mu \mathrm{A}$, respectively. In the case of unetched CIA-TENG, those were $149 \mathrm{~V}$ and $126.4 \mu \mathrm{A}$, respectively (Additional file 1: Fig. S3). These triboelectric properties are significantly inferior to those of corresponding references with the etched dielectric film (Fig. 2). The improved PTFE surface roughness increases the contact surface area to the Al top electrode, resulting in more triboelectric charges on the PTFE surface. It is noticeable that the degree of short-circuit current enhancement is larger than that of open-circuit voltage enhancement, which can be explained by the following equation: [43]

$$
V=-\frac{Q}{S_{e f f} \epsilon_{0}}\left(d_{0}+x(t)\right)+\frac{\sigma_{\text {tribo }} x(t)}{\epsilon_{0}}
$$

where, $S_{e f f}, d_{0}, \sigma_{t r i b o}$, and $x(t)$ are the effective contact area of the dielectric film to the electrode, the effective dielectric thickness constant $(\mathrm{d} / \varepsilon$, d: dielectric thickness, $\varepsilon$ : relative dielectric constant), triboelectric charges, and the separation distance depending on the time, respectively. In the open-circuit condition, the transferred charges (Q) between electrodes are 0 ; therefore, the $\mathrm{V}_{\mathrm{oc}}$ is affected by only $\sigma_{\text {tribo }}$ and thus linearly proportional to only $\sigma_{\text {tribo }}$ by Eq. (1). At a short-circuit condition, $I_{\text {sc }}$ is given by: [43]

$$
I_{s c}=\frac{d Q_{s c}}{d t}=-\frac{S_{e f f} \sigma_{t r i b o} d_{0}}{\left(d_{0}+x(t)\right)^{2}} \frac{d x}{d t}=\frac{S_{e f f} \sigma_{t r i b o} d_{0} v(t)}{\left(d_{0}+x(t)\right)^{2}}
$$

where $v(t)$ is the fluttering speed of the dielectric film. According to Eq. (2), $\mathrm{I}_{\mathrm{sc}}$ is linearly proportional to the multiplied value of $S_{\text {eff }}$ and $\sigma_{\text {tribo }}$. The etching process increased the surface roughness, inducing more contact surface area during electrification, and more triboelectric charges on the PTFE surface, resulting in the higher increment of $\mathrm{I}_{\mathrm{sc}}$ in comparison to the $\mathrm{V}_{\mathrm{oc}}$ increment. 


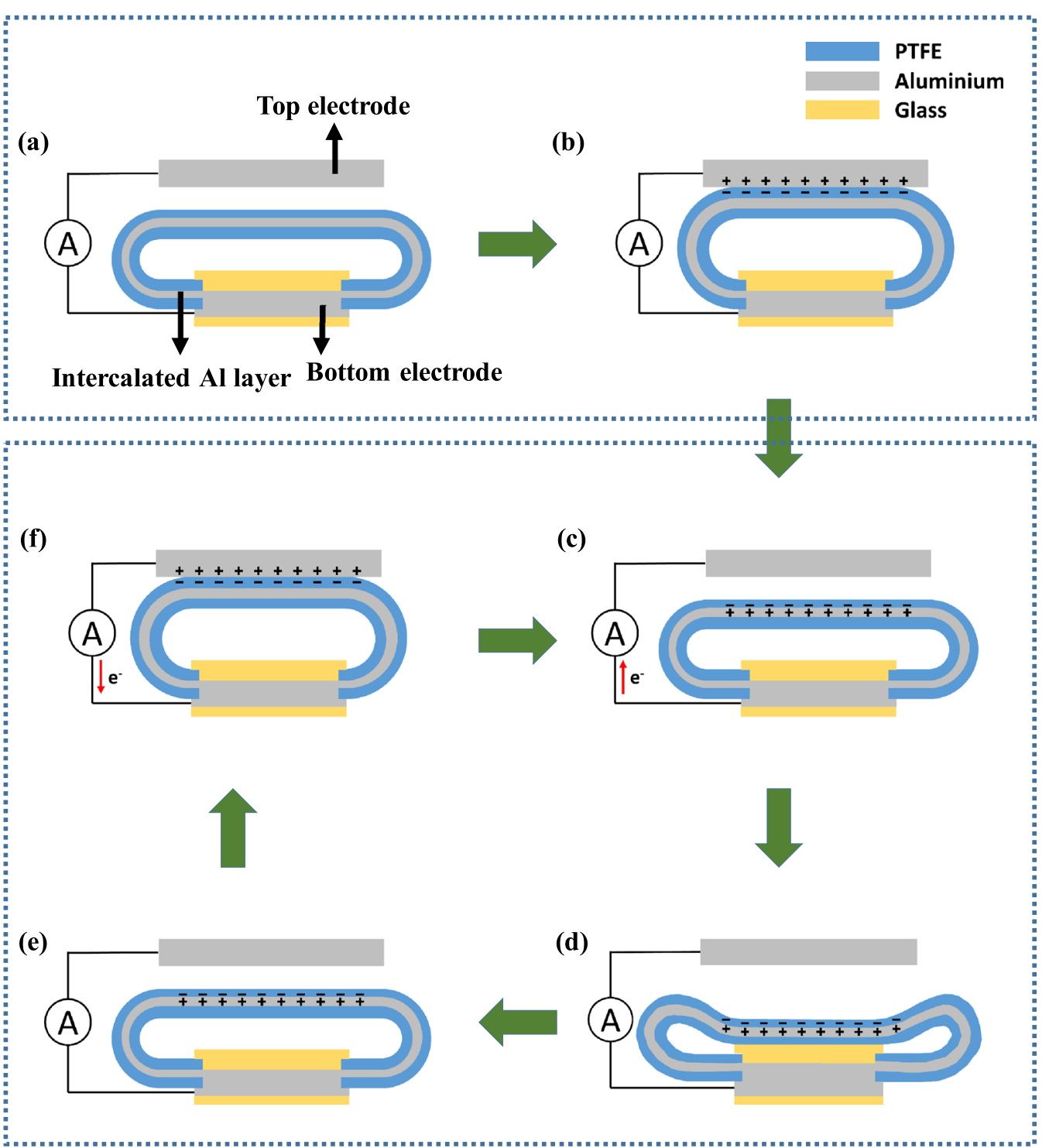

Fig. 3 Operating mechanisms of CIA-TENG; $\mathbf{a}$, $\mathbf{b}$ initial electrification step and $\mathbf{c}-\mathbf{f}$ repetitive triboelectric step

\subsection{Operating mechanism of the C-TENG and CIA-TENG}

To understand the effect of the intercalated Al layer, the operating mechanism of the C-TENG and CIA-TENG was analyzed as depicted in Additional file 1: Fig. S4 and Fig. 3. Two primary mechanisms are used for the operation of the wind-driven TENG. The first mechanism is the electrification of the dielectric film through the contact between the two triboelectric materials. The second step is electrostatic induction, in which the free electrons at the electrode surface are repulsed by the negative triboelectric charges sitting on the dielectric film when it approaches the electrode. As a result, an equal amount of positive charges remains on the electrode surface.

In the C-TENG, when the C-film contacts the Al top electrode, negative triboelectric charges are accumulated on the surface of the C-film by withdrawing free electrons from the Al top electrode (Additional file 1: Fig. S4a and b). During the initial electrification cycles, triboelectric charges are accumulated on both surfaces of the $\mathrm{C}$-film and $\mathrm{Al}$ top electrode. When detaching, as the distance between the $\mathrm{C}$-film and Al top electrode increases, the electrostatic induction to the Al top electrode by the 
negative charges of the C-film is gradually weakened, resulting in a flow of electrons from the bottom to the top electrode (Additional file 1: Fig. S4c) until the electrostatic induction is no longer active on the surface of the Al top electrode (Additional file 1: Fig. S4d). On the contrary, when the negatively charged C-film approaches the $\mathrm{Al}$ top electrode, electrons move from the top to the bottom electrode, because Coulomb repulsion occurs between the negative triboelectric charges of the C-film and the free electrons of the $\mathrm{Al}$ top electrode (Additional file 1: Fig. S4e and f).

The same mechanism is applicable to the CIA-TENG during the initial few cycles, giving rise to negative triboelectric charges on the CIA-film surface (Fig. $3 a$ and $b$ ). As soon as the negatively charged CIA-film is detached from the $\mathrm{Al}$ top electrode, more electrons move from the bottom to the top electrode than those of the C-TENG because sudden electrostatic equilibrium was generated at the interface between the negatively charged CIA-film and the intercalated $\mathrm{Al}$ layer, generating positive charges on the intercalated $\mathrm{Al}$ surface (Fig. 3c). Concurrently, the Coulomb repulsion between the triboelectric electrons of the CIA-film and free electrons of the intercalated Al layer occurs. These two combined effects (electrostatic induction and Coulomb repulsion) induce more electrons' flow to the $\mathrm{Al}$ top electrode until electrostatic equilibrium is reached at which the net current is zero (Fig. 3d and e). Therefore, the intercalated $\mathrm{Al}$ layer within the PTFE film generates more electricity in comparison to the C-TENG. When the CIA-film touches the top electrode in the next oscillation, electrons immediately move from the top electrode to the intercalated Al layer to maintain electrostatic equilibrium at the interface between the CIA-film and $\mathrm{Al}$ top electrode (Fig. 3f). This triboelectric mechanism continues in the following oscillation. A detailed explanation of the effect of intercalated Al layer within the PTFE film was given in a previously reported paper [44].

\subsection{Effect of gap, wind speed, and wind direction on the triboelectric performances of the CIA-TENG}

The CIA-TENG was operated under various conditions to check the effect of the gap, wind speed, and wind direction. Figure $4 \mathrm{a}$ and $\mathrm{b}$ show the $\mathrm{V}_{\mathrm{oc}}$ and $\mathrm{I}_{\mathrm{sc}}$ of CIATENG measured at various gaps. The $\mathrm{V}_{\mathrm{oc}}$ and $\mathrm{I}_{\mathrm{sc}}$ were $188,233,155$, and $120 \mathrm{~V}$ and $271,348,205$, and $122 \mu \mathrm{A}$ at the gap of $3,4,5$, and $6 \mathrm{~mm}$, respectively, revealing the maximum triboelectric performance at a gap of $4 \mathrm{~mm}$. The fluttering frequencies of CIA-film were 260, 306, 242 , and $204 \mathrm{~Hz}$ at the corresponding gaps of 3, 4, 5, and
$6 \mathrm{~mm}$ at a wind speed of $9 \mathrm{~m} \mathrm{~s}^{-1}$ (Fig. 4c). The fluttering frequency was calculated by counting the number of $I_{\mathrm{sc}}$ peaks at an interval of $0.05 \mathrm{~s}$ (Additional file 1: Fig. S5). The highest fluttering frequency of $306 \mathrm{~Hz}$ was measured at a gap of $4 \mathrm{~mm}$ at which the best wind-driven triboelectric performance was demonstrated. The narrower gap $(3 \mathrm{~mm})$ disturbed the fluttering of the CIA-film, resulting in a lower fluttering frequency, which implies that the dielectric film impacts the $\mathrm{Al}$ top electrode with a lower speed of $v(t)$, rendering a drop in $\mathrm{I}_{\mathrm{sc}}$ according to Eq. (2). The $V_{o c}, I_{s c}$ and fluttering frequency were measured at various wind speeds (Fig. $4 \mathrm{~d}-\mathrm{f}$ ). As expected, the fluttering frequency increased with the wind speed; thus, both $\mathrm{V}_{\mathrm{oc}}$ and $\mathrm{I}_{\mathrm{sc}}$ increased with wind speed.

To verify that the CIA-TENG enables energy harvesting in all wind directions, the triboelectric performance was measured depending on the wind angles (Fig. $4 \mathrm{~g}$ and h). The wind angle $(\theta)$ was defined as illustrated in Fig. 4i. The maximum $\mathrm{V}_{\mathrm{oc}}$ and $\mathrm{I}_{\mathrm{sc}}$ were revealed at $0,90,180$, and $270^{\circ}$, where the wind blows vertically to the CIAfilm plane. In contrast, the minimum $\mathrm{V}_{\mathrm{oc}}$ and $\mathrm{I}_{\mathrm{sc}}$, which were approximately 58 and $32 \%$ of the maximum $\mathrm{V}_{\text {oc }}$ and $I_{\mathrm{sc}}$, respectively, were recorded at $45,135,225$, and $305^{\circ}$, where the wind blows diagonally to the void between the CIA-films. These results indicate that wind energy can be harvested regardless of the wind direction with the crossshaped dielectric film.

\subsection{Application of the CIA-TENG}

A continuous operation was performed at a wind speed of $9 \mathrm{~m} \mathrm{~s}^{-1}$ to test the durability of the CIA-TENG. Figure $5 \mathrm{a}$ shows that the $\mathrm{V}_{\mathrm{oc}}$ was maintained for approximately $1650 \mathrm{~s}$, corresponding to 500,000 fluttering cycles, and gradually decreased. For practical application, the CIATENG was connected to a bridge rectifier to convert the AC to a DC signal, and 25 light-emitting diodes (LED) bulbs in series (Fig. 5b). The resulting maximum $\mathrm{V}_{\text {oc }}$ of $120.4 \mathrm{~V}$ and $\mathrm{I}_{\mathrm{sc}}$ of $237.6 \mu \mathrm{A}$ were 48 and $32 \%$ lower than those of the AC signal, respectively (Fig. $5 \mathrm{c}$ and d). The CIA-TENG supplied a stable power to 25 LEDs to light "LED" characters (Fig. 5e and Additional file 3: Movie S2).

\section{Conclusions}

In this study, wind-driven C-TENG and CIA-TENG were fabricated and their triboelectric performances were compared. Cross-shaped dielectric films bent in four directions were proposed to harvest the wind energy regardless of wind directions. By inserting an $\mathrm{Al}$ layer within the dielectric film, the combined effects of electrostatic equilibrium and Coulomb repulsion between the 
(a)

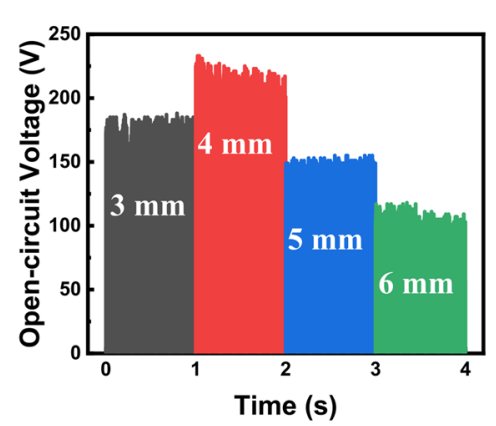

(d)

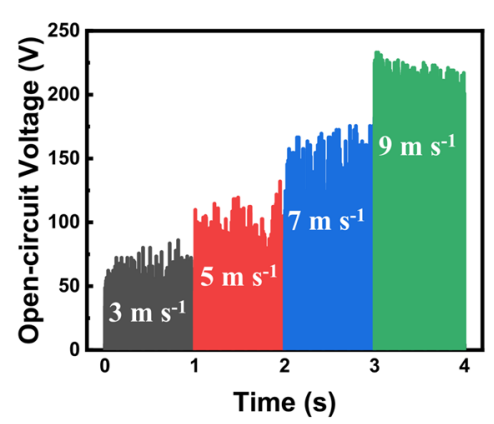

(g)

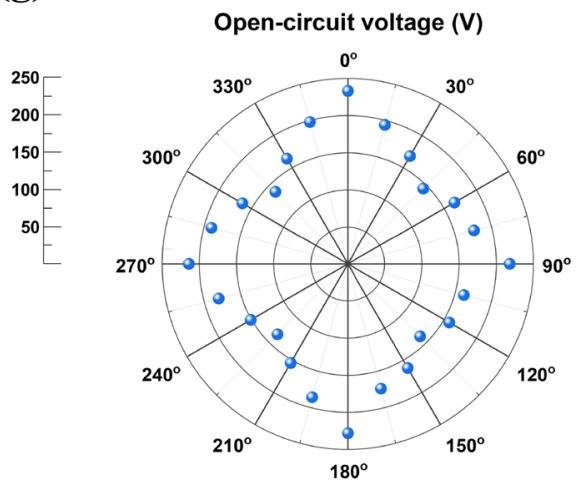

(b)

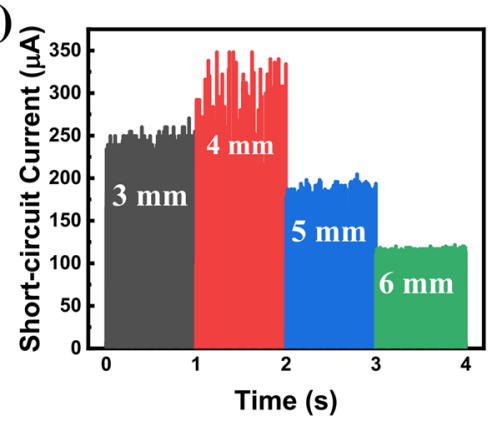

(e)

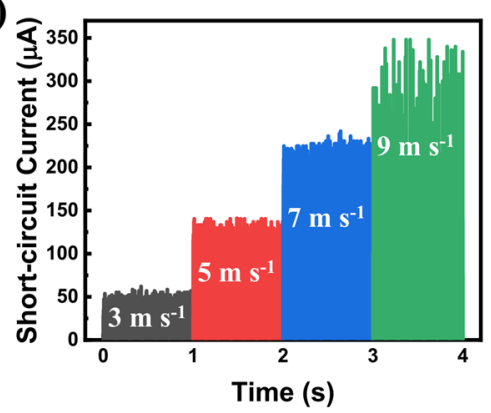

(h)

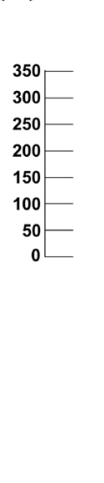

(c)

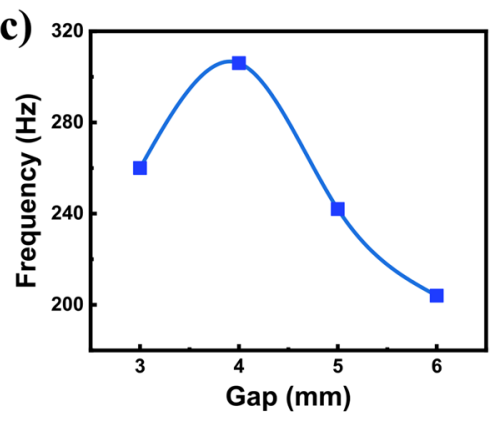

(f)

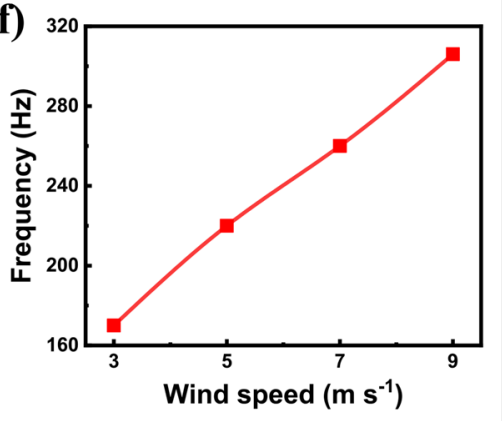

(i)

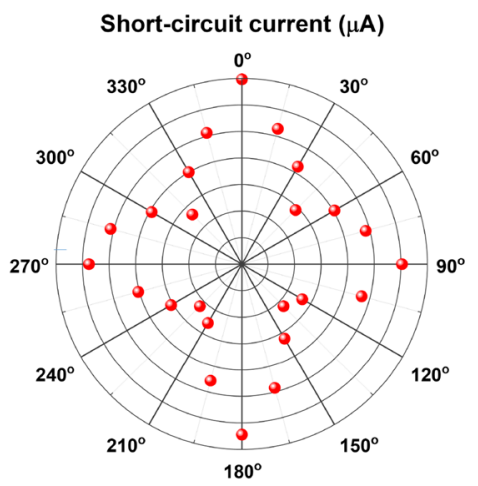

$\theta$ : Wind angle

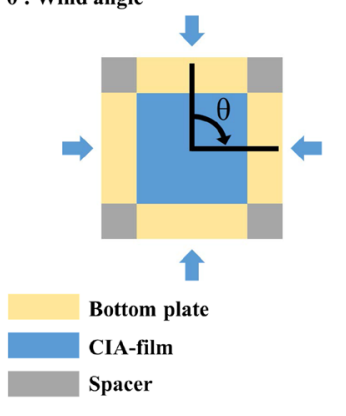

Fig. 4 Triboelectric performances of the CIA-TENG at various a-c gaps, $\mathbf{d}-\mathbf{f}$ wind speeds, and $\mathbf{g}-\mathbf{i}$ wind directions shown from the top view of CIA-TENG

CIA-film and the intercalated Al layer generated more electrons for the triboelectric performance. When comparing the triboelectric performances of the C-TENG and CIA-TENG, the $\mathrm{V}_{\mathrm{oc}}, \mathrm{I}_{\mathrm{sc}}$, and output power density of the CIA-TENG were 1.52, 6.72, and 20 times higher than those of the C-TENG, respectively. The CIA-TENG could harvest energies sustainably by the wind blowing in arbitrary directions; the CIA-TENG could be installed and supply an auxiliary power to the vehicles moving in arbitrary ways. 
(a)

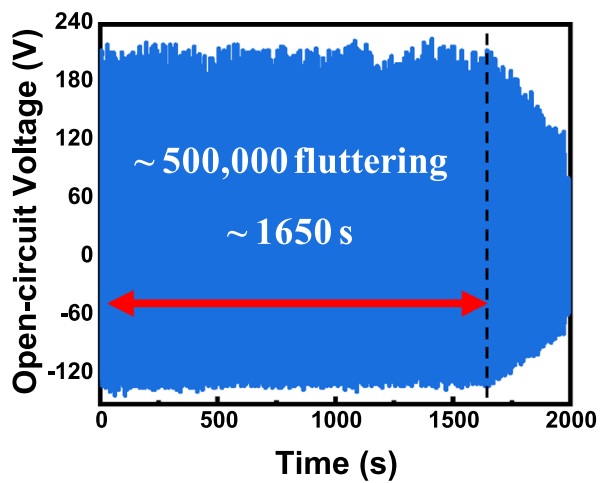

(c)

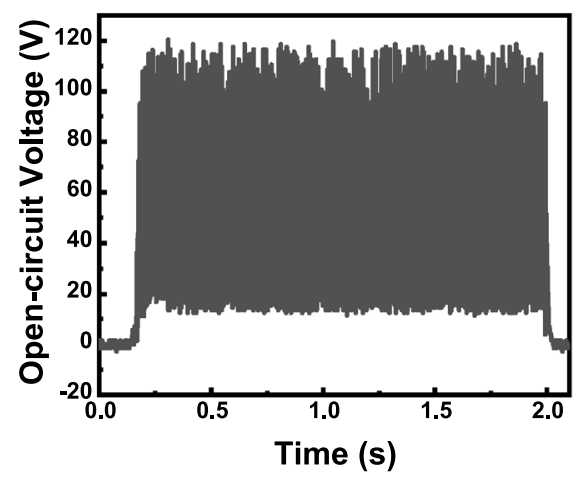

(e) (b)

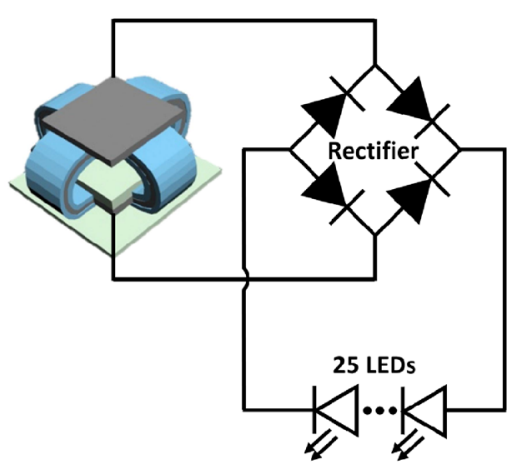

(d)

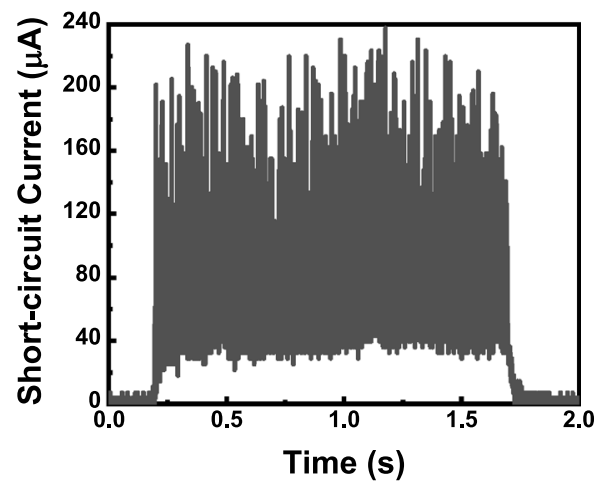

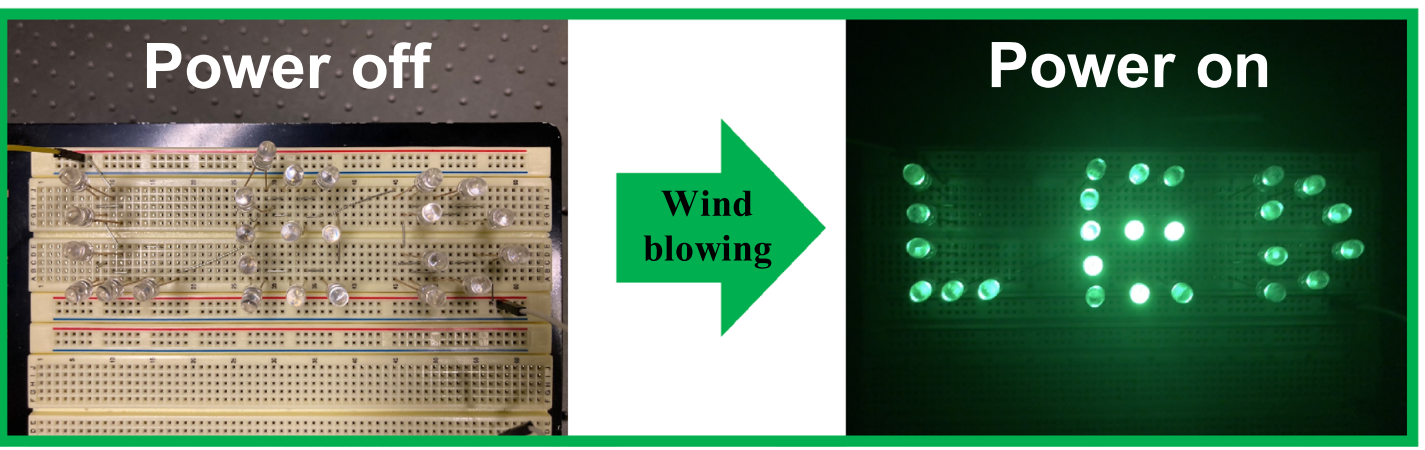

Fig. 5 a The change of $\mathrm{V}_{\text {oc }}$ of the CIA-TENG at a wind speed of $9 \mathrm{~m} \mathrm{~s}^{-1}$ for 500,000 fluttering cycles. b A schematic circuit diagram for powering 25 LEDs and $\mathbf{c}$, $\mathbf{d}$ the triboelectric performances of the CIA-TENG with a bridge rectifier in series. e Snapshot of the lit "LED" characters composed of 25 LEDs, powering by the CIA-TENG

\section{Abbreviations}

TENG: Triboelectric nanogenerator; Al: Aluminum; $V_{0}$ : Open-circuit voltage; $\mathrm{I}_{\text {sc }}$ : Short-circuit current; loT: Internet of Thing; C-TENG: Wind-driven TENG structure with a cross-shaped dielectric film bent in four directions; PTFE: Polytetrafluoroethylene; CIA-TENG: C-TENG in which an Al layer is intercalated within a PTFE film; F: Fluorine; ICP-RIE: Inductively coupled plasma-reactive ion etching; C-film: Cross-shaped PTFE film; CIA-film: C-film having the intercalated Al layer; RMS: Root-mean-square; $V_{\text {rms }}$ : RMS voltage; $P_{\text {avg }}$ : Average output power; LED: Light-emitting diode.

\section{Supplementary Information}

The online version contains supplementary material available at https://doi. org/10.1186/s40580-021-00276-5.

Additional file 1: Fig. S1. AFM images of PTFE films (a) before and (b) after RIE etching. Fig. S2. (a) Maximum and RMS output voltage, and (b) average power vs. external resistance. Fig. S3. Voc and Isc of $(a, b)$ the C-TENG and $(c, d)$ the CIA-TENG using the unetched PTFE film at a wind speed of $9 \mathrm{~m} \mathrm{~s}^{-1}$. Fig. S4. Operating mechanisms of C-TENG; $(a, b)$ initial 
electrification step and (c-f) repetitive triboelectric step. Fig. S5. Comparison of Isc at different (a) gaps and (b) wind speeds (interval: $0.05 \mathrm{~s}$.

Additional file 2: Movie S1. Vertical vibration of the CIA-TENG at a wind speed of $9 \mathrm{~m} \mathrm{~s}^{-1}$.

Additional file 3: Movie S2. Demonstration of powering 25 LEDs by a CIA-TENG.

\section{Acknowledgements}

This research was supported by the Basic Science Research Program (NRF2019R1A2B5B01070640) of the National Research Foundation of Korea funded by the Ministry Science and ICT and the GIST Research Institute (GRI) grant funded by the GIST.

\section{Authors' contributions}

YS, SC, and GYJ designed the concept and the required experiments. YS and SC prepared the materials, conducted the tests of triboelectric performance and SH analyzed the obtained data. YS and SC fabricated the devices, and performed the $\mathrm{V}_{\text {oc }}$ and Isc measurements and prepared the figures. SH scanned the surface of materials and measured RMS roughness by AFM. YS, SC, and GYJ interpreted the data and wrote the manuscript. All authors discussed the results and commented on the manuscript.

\section{Funding}

This work was supported by the National Research Foundation of Korea (Basic Science Research Program (NRF-2019R1A2B5B01070640) funded by the Ministry of Science and ICT, and the GIST Research Institute (GRI) grant funded by the GIST in 2020.

\section{Availability of data and material}

The datasets used and/or analyzed during the current study are available from the corresponding author on reasonable request.

\section{Declarations}

\section{Competing interests}

The authors declare that they have no competing interests.

Received: 29 April 2021 Accepted: 12 August 2021

Published online: 02 September 2021

\section{References}

1. J. Gubbi, R. Buyya, S. Marusic et al., Internet of Things (IoT): a vision, architectural elements, and future directions. Future Gener. Comput. Syst. 29 1645-1660 (2013). https://doi.org/10.1016/j.future.2013.01.010

2. L. Atzori, A. lera, G. Morabito, The Internet of Things: a survey. Comput. Netw. 54, 2787-2805 (2010). https://doi.org/10.1016/j.comnet.2010.05. 010

3. A. Al-Fuqaha, M. Guizani, M. Mohammadi et al., Internet of Things: a survey on enabling technologies, protocols, and applications. IEEE Commun. Surv. Tutor. 17, 2347-2376 (2015). https://doi.org/10.1109/COMST.2015. 2444095

4. B. Pelaz, C. Alexiou, R.A. Alvarez-Puebla et al., Diverse applications of nanomedicine. ACS Nano 11, 2313-2381 (2017). https://doi.org/10.1021/ acsnano.6b06040

5. O.D. Lara, M.A. Labrador, A survey on human activity recognition using wearable sensors. IEEE Commun. Surv. Tutor. 15, 1192-1209 (2013). https://doi.org/10.1109/SURV.2012.110112.00192

6. J. Heikenfeld, A. Jajack, J. Rogers et al., Wearable sensors: modalities, challenges, and prospects. Lab Chip 18, 217-248 (2018). https://doi.org/10. 1039/c7lc00914c

7. J.R. Sempionatto, T. Nakagawa, A. Pavinatto et al., Eyeglasses based wireless electrolyte and metabolite sensor platform. Lab Chip 17, 1834-1842 (2017). https://doi.org/10.1039/c7lc00192d
8. S.T. Perrault, E. Lecolinet, J. Eagan et al., Watchlt: Simple Gestures and Eyes-free Interaction for Wristwatches and Bracelets. In: CHI Proceedings. 2013. p. 1451-1460. https://doi.org/10.1145/2470654.2466192

9. N.S. Shenck, J.A. Paradiso, Energy scavenging with shoe-mounted pieozoelectrics. IEEE Micro 21, 30-42 (2001). https://doi.org/10.1109/40.928763

10. W. Xu, J. Wang, F. Ding et al., Lithium metal anodes for rechargeable batteries. Energy Environ. Sci. 7, 513-537 (2014). https://doi.org/10.1039/ c3ee40795k

11. G. Zhou, F. Li, H.M. Cheng, Progress in flexible lithium batteries and future prospects. Energy Environ. Sci. 7, 1307-1338 (2014). https://doi.org/10. 1039/c3ee43182g

12. X.-B. Cheng, T.-Z. Hou, R. Zhang et al., Dendrite-free lithium deposition induced by uniformly distributed lithium lons for efficient lithium metal batteries. Adv. Mater. 28, 2888-2895 (2016). https://doi.org/10.1002/ adma.201506124

13. M. Banerjee, J. Lee, K.-K.R. Choo, A blockchain future for internet of things security: a position paper. Digit. Commun. Netw. 4, 149-160 (2018). https://doi.org/10.1016/j.dcan.2017.10.006

14. C. Perera, C.H. Liu, The emerging internet of things marketplace from an industrial perspective: a survey. IEEE Trans. Emerg. Topics Comput. 3, 585-598 (2015). https://doi.org/10.1109/TETC.2015.2390034

15. J. Chen, K. Hu, Y. Sun et al., Narrowband internet of things: Implementations and applications. IEEE Internet Things J. 4, 2309-2314 (2017). https://doi.org/10.1109/JOT.2017.2764475

16. R. Montella, S. Kosta, I. Foster, DYNAMO: Distributed leisure Yacht-carried sensor-Network for Atmosphere and Marine data crOwdsourcing applications. In: IEEE internetional conference on cloud engineering. 2018. p.333-339. https://doi.org/10.1109/IC2E.2018.00064

17. R. Venkatasubramanian, E. Siivola, T. Colpitts et al., Thin-film thermoelec tric devices with high room-temperature figures of merit. Nature $\mathbf{4 1 3}$ 597-602 (2001). https://doi.org/10.1038/35098012

18. A. Kojima, K. Teshima, Y. Shirai et al., Organometal halide perovskites as visible-light sensitizers for photovoltaic cells. J. Am. Chem. Soc. 131, 6050-6051 (2009). https://doi.org/10.1021/ja809598r

19. T.Y. Kim, S.K. Kim, S.-W. Kim, Application of ferroelectric materials for improving output power of energy harvesters. Nano Convergence $\mathbf{5}$, 5-30 (2018). https://doi.org/10.1186/s40580-018-0163-0

20. B.E. Hardin, H.J. Snaith, M.D. McGehee, The renaissance of dye-sensitized solar cells. Nat. Photonics 6, 162-169 (2012). https://doi.org/10.1038/ nphoton.2012.22

21. C. Wu, A.C. Wang, W. Ding et al., Triboelectric nanogenerator: a foundation of the energy for the new era. Adv. Energy Mater. 9, 1802906 (2019). https://doi.org/10.1002/aenm.201802906

22. Y. Yang, G. Zhu, H. Zhang et al., Triboelectric nanogenerator for harvesting wind energy and as self-powered wind vector sensor system. ACS Nano 7, 9461-9468 (2013). https://doi.org/10.1021/nn4043157

23. C. Chang, V.H. Tran, J. Wang et al., Direct-write piezoelectric polymeric nanogenerator with high energy conversion efficiency. Nano Lett. 10 726-731 (2010). https://doi.org/10.1021/nl9040719

24. B. Honig, A. Nicholls, Classical electrostatics in biology and chemistry. Science 268, 1144-1149 (1995). https://doi.org/10.1126/science.7761829

25. K.-I. Park, S. Xu, Y. Liu et al., Piezoelectric BaTiO3 thin film nanogenerator on plastic substrates. Nano Lett. 10, 4939-4943 (2010). https://doi.org/10. 1021/nl102959k

26. Y. Gao, Z.L. Wang, "Electrostatic potential in a bent piezoelectric nanowire The fundamental theory of nanogenerator and nanopiezotronics. Nano Lett. 7, 2499-2505 (2007). https://doi.org/10.1021/nl071310j

27. M. Lee, C.-Y. Chen, S. Wang et al., A hybrid piezoelectric structure for wearable nanogenerators. Adv. Mater. 24, 1759-1764 (2012). https://doi.org/ 10.1002/adma.201200150

28. M.-P. Lu, J. Song, M.-Y. Lu et al., Piezoelectric nanogenerator using p-type ZnO nanowire arrays. Nano Lett. 9, 1223-1227 (2009). https://doi.org/10. $1021 / n 1900115 y$

29. W. Wang, J. Xu, H. Zheng et al., A spring-assisted hybrid triboelectricelectromagnetic nanogenerator for harvesting low-frequency vibration energy and creating a self-powered security system. Nanoscale 10 14747-14754 (2018). https://doi.org/10.1039/c8nr04276d

30. B. Chen, Y. Yang, Z.L. Wang, Scavenging wind energy by triboelectric nanogenerators. Adv. Energy Mater. 8, 1702649 (2018). https://doi.org/10. 1002/aenm.201702649 
31. L. Lin, S. Wang, S. Niu et al., Noncontact free-rotating disk triboelectric nanogenerator as a sustainable energy harvester and self-powered mechanical sensor. ACS Appl. Mater. Interfaces 6, 3031-3038 (2014). https://doi.org/10.1021/am405637s

32. J. Hu, X. Pu, H. Yang et al., A flutter-effect-based triboelectric nanogenerator for breeze energy collection from arbitrary directions and self-powered wind speed sensor. Nano Res. 12, 3018-3023 (2019). https://doi.org/ 10.1007/s12274-019-2545-y

33. L. Zhang, B. Meng, Y. Xia et al., Galloping triboelectric nanogenerator for energy harvesting under low wind speed. Nano Energy 70, 104477 (2020). https://doi.org/10.1016/j.nanoen.2020.104477

34. H. Kim, Q. Zhou, D. Kim et al., Flow-induced snap-through triboelectric nanogenerator. Nano Energy 68, 104379 (2020). https://doi.org/10.1016/j. nanoen.2019.104379

35. N.Y.W. Zaw, H. Roh, I. Kim et al., Omnidirectional triboelectric nanogenerator operated by weak wind towards a self-powered anemoscope. Micromachines 11, 414 (2020). https://doi.org/10.3390/mi1 1040414

36. L. Zhang, B. Zhang, J. Chen et al., Lawn structured triboelectric nanogenerators for scavenging sweeping wind energy on rooftops. Adv. Mater. 28, 1650-1656 (2016). https://doi.org/10.1002/adma.201504462

37. W. Sun, Z. Ding, Z. Qin et al., Wind energy harvesting based on fluttering double-flag type triboelectric nanogenerators. Nano Energy 70, 104526 (2020). https://doi.org/10.1016/j.nanoen.2020.104526

38. A.N. Ravichandran, C. Calmes, J.R. Serres et al., Compact and high performance wind actuated venturi triboelectric energy harvester. Nano Energy 62,449-457 (2019). https://doi.org/10.1016/j.nanoen.2019.05.053
39. S. Thiele, K. Arzenbacher, T. Gissibl et al., 3D-printed eagle eye: compound microlens system for foveated imaging. Sci. Adv. 3, 1602655 (2017). https://doi.org/10.1126/sciadv.1602655

40. A. Mairaj, A. Baba, A.Y. Javaid, Application specific drone simulators: recent advances and challenges. Simul. Model. Pract. Theory 94, 100-117 (2020). https://doi.org/10.1016/j.simpat.2019.01.004

41. K. Dorling, J. Heinrichs, G.G. Messier et al., Vehicle routing problem for drone delivery. IEEE Trans. Syst. Man Cybern. Syst. 47, 70-85 (2017). https://doi.org/10.1109/TSMC.2016.2582745

42. M. Mozaffari, W. Saad, M. Bennis et al., Wireless communication using unmanned aerial vehicles (UAVs): optimal transport theory for hover time optimization. IEEE Trans. Wireless Commun. 16, 8052-8066 (2017). https://doi.org/10.1021/ja809598r

43. S. Niu, S. Wang, L. Lin,et al, Theoretical study of contact-mode triboelectric nanogenerators as an effective power source. Energy Environ. Sci. 6, 3576-3583 (2013). https://doi.org/10.1039/c3ee42571a

44. S. Cho, Y. Shin, J. Choi et al., Triboelectric nanogenerator based on intercalated Al layer within fluttering dielectric film. Nano Energy 77, 105184 (2020). https://doi.org/10.1016/j.nanoen.2020.105184

\section{Publisher's Note}

Springer Nature remains neutral with regard to jurisdictional claims in published maps and institutional affiliations.

\section{Submit your manuscript to a SpringerOpen ${ }^{\odot}$ journal and benefit from:}

- Convenient online submission

- Rigorous peer review

- Open access: articles freely available online

- High visibility within the field

- Retaining the copyright to your article

Submit your next manuscript at $\boldsymbol{\nabla}$ springeropen.com 